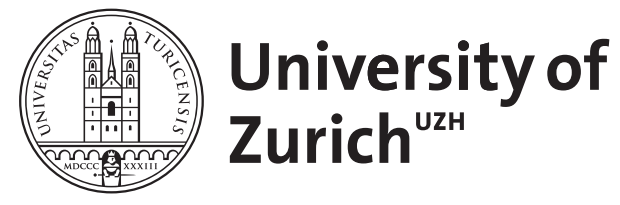

\title{
Clinical MR in the year 2010
}

\author{
von Schulthess, G K
}

\begin{abstract}
This article tries to look into the future of magnetic resonance imaging. Deducing from six rules, it pinpoints the most likely developments of this imaging technique
\end{abstract}

DOI: https://doi.org/10.1007/bf02594591

Posted at the Zurich Open Repository and Archive, University of Zurich ZORA URL: https://doi.org/10.5167/uzh-156319

Journal Article

Published Version

Originally published at: von Schulthess, G K (1999). Clinical MR in the year 2010. Magma, 8(3):133-145. DOI: https://doi.org/10.1007/bf02594591 


\title{
Clinical MR in the year $2010^{\text {th }}$
}

\author{
G.K. von Schulthess * \\ Universitätsspital Zurich, Nuklearmedizin, Rämistraße 100, CH-8029 Zurich. Switzerland
}

\begin{abstract}
This article tries to look into the future of magnetic resonance imaging. Deducing from six rules, it pinpoints the most likely developments of this imaging technique. 1999 Elsevier Science B.V. All rights reserved.
\end{abstract}

Ke?worls: Magnetic resonance imaging; Contrast agents; MR angiography; PET

\section{Introduction}

This overview tries to outline some basic principles to identify good strategies to look for future successful applications of MR imaging in the clinical environment, and define where MR may be in the year 2010 . The author is aware of the fact that if fate will permit it, he will still be active in his profession and MR by 2010 , therefore jeopardizing his reputation. Nevertheless, he thinks that challenging the readers and stimulating their own rebuttals to his theses will make them look at future opportunities more critically, and thus potentially define their future projects such that they will have a higher chance to proceed.

Predicting the future is difficult, a commonplace statement, and in order to prevent the reader from skipping this article and go to the next contribution, the author obviously has to start with a disclaimer. The thoughts presented here are a digest of discussions with many friends, who have shaped this author's thoughts. However, the responsibility for the statements are all his. All omissions and inclusions, all names which are improperly mentioned and not mentioned are the author's responsibilities and he is aware of the fact that many brilliant researchers will be unhappy with his statements, and some may be happy. This contribution

\footnotetext{
* This article is based on the Crystal Ball Lecture held at the International Society of Magnetic Resonance in Medicine (ISMRM) in Sydney, 20 April 1998, and the Award Lecture after receiving the European Magnetic Resonance Award 1998 for Medical Sciences of the EMRF Foundation at Fuschl (Austria) on 5 June 1998

* Tel.: + 41-1-255-2944; fax: + 41-1-255-4428.

E-mail address: vonschulthess@dmr.usz.ch (G.K. von Schuithess)
}

is intended to stimulate discussion and so the author will not try to say anything just in order not to be wrong or to offend, but will try to stick his head out.

The history of Magnetic Resonance in Medicine, is such that there always have been heated but stimulating discussions, including fist fights by protagonists of 1.5 and $0.5 \mathrm{~T}$ systems in the early MR days.

This contribution will be divided into four parts; first an introduction, second a part where the stage is set for the predictions, third the discussion of the predictions proper and fourth the conclusions and suggested investment strategies. While predicting the future is difficult, the author will be in good company when making mistakes: in fact, Lord Kelvin described the discovery of X-rays a hoax. Why is it so difficult to predict the future? Our sciences evolve steadily, and steady development is predictable by linear extrapolation. But history in any field of knowledge shows us that there are moments when the old ways of thinking: the old paradigms no longer are adequate guidelines. 'Revolutionary' changes in paradigms occur, as Kuhn stated [1]. The latter make the future unpredictable because suddenly a completely new approach changes the practice of some science. I might add that these paradigmatic changes also make life exciting.

In such moments, new ways have to be found which represent complete shifts in these paradigms. Examples are the discovery of quantum mechanics and the special theory of relativity. But the introduction of $\mathrm{X}$-rays is also a dramatic change in the paradigms of medical diagnosis: suddenly it became possible to diagnose chest disease and bone disease with a high degree of accuracy. Other paradigmatic changes in imaging are the 
introduction of angiography, metabolic imaging with radionuclides and with $\mathrm{PET}$, and cross-sectional imaging. The discovery of NMR certainly was also such a revolution. Was the introduction of $M R$ imaging into clinical medicine? In many places MR only added incremental benefits compared to $\mathrm{CT}$, so the introduction of CT was maybe more of a revolution. Still, there are important aspects in MRI which can be called 'revolutionary' such as multiplanar imaging and other 'revolutionary' aspects of MR to be discussed.

To exemplify one novel aspect of MR imaging, the multiplanar imaging capabilities of MR, Fig. 1 shows a somewhat historical image, namely that of the brain of Richard R. Ernst. Chemistry Nobel Laureate of 1991 for his eminent contributions to NMR. There is an interesting note regarding this image: morphology does not tell you anything about the intelligence of this individual.

We conclude, that while it is important to be an enthusiast in the field one is working in, one should keep one's mind open and be fair to competing techniques. Decisive revolutions may happen in a different field from that one is working in.

\section{The approach to the problem}

At the outset of this presentation of predicting the status of clinical MR imaging in the year 2010 the key question to be asked is thus: is there a rational approach to predict the future of clinical MR? This author, in fact, believes that one can establish some guidelines where to look for future important developments. This may serve as sort of a flashlight to guide us in the dark of choosing future projects to work on.

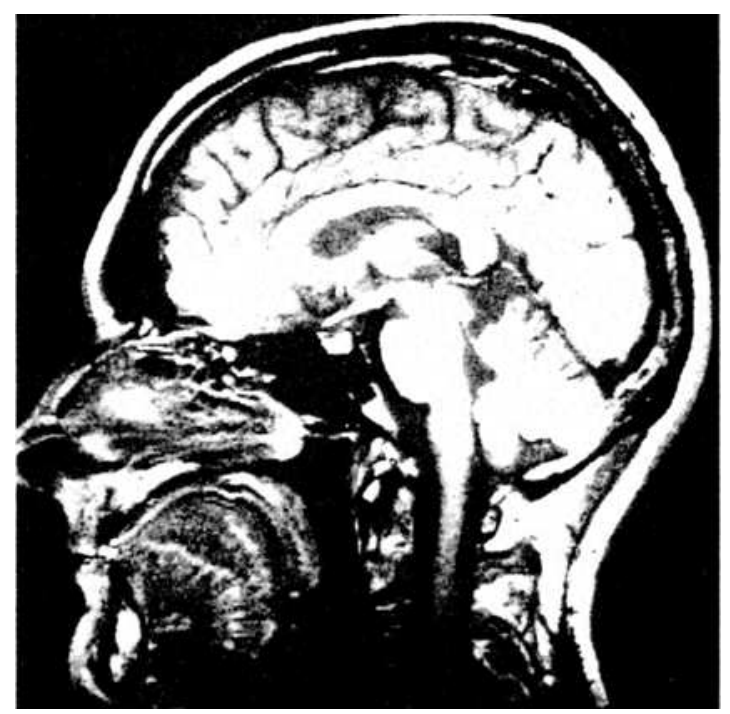

Fig. 1. Sagittal Tl-weighted image (1986) of Professor Richard R. Ernst, Nobel Laureate in Chemistry 1991 and inventor of Fourier transform imaging and spectroscopy.
What are the medical imaging needs? Where are major unsolved questions in diagnosis and disease management, with imaging playing a vital role?

Three areas come to my mind, where progress will be very important for disease control: affective disorders, atherosclerotic cardiovascular disease and tumor imaging. Characterization of schizophrenia, dementia and depression has been difficult and there are guarded hopes that receptor imaging may eventually help to unravel some of the mysteries of these disease processes. There remain vast uncharted territories, where imaging may be relevant, but there is little hope that the sensitivity of MR will eventually reach a level where receptors can be imaged. This will likely remain the domain of nuclear imaging techniques. In the cardiovascular system we are pretty good in imaging. Blind spots are the non-invasive imaging of atherosclerotic plaques, the early detection of stroke and high resolution ischemia imaging. While imaging of molecular processes using MR might also be interesting, this is too far away to be considered here, cardiac MR spectroscopy is easily the greatest challenge in MR. Tumor imaging for staging purposes is still suboptimal in many metastatic tumors. Higher sensitivity and specificity imaging is very desirable and metabolic imaging is of great hope in this area. Tumor therapy is crude, better methods to monitor tumor development under therapy are dearly needed.

Combining the list of where imaging is needed and the list of the number of measurable and changeable MR parameters, the future for MR looks bright indeed, and one could stop here and say, MR will thrive on its 'random walk' in the dimensions comprising all the changeable MR parameters. There will be one billion more papers on $M R$ and the annual meeting of the ISMRM will have replaced RSNA by 2010 , since nobody uses any other imaging modality any more, etc. On the other hand, a critical appraisal of the breakthroughs in MR which have led to substantial changes in clinical disease management shows a somewhat different picture.

The introduction of spin-echo and gradient-echo sequences and the introduction of contrast media have been basic, and led to MR imaging becoming the premier imaging modality of the brain and the musculoskeletal system. Introduction of flow quantification has given us the gold standard tool for flow measurements, although the relevance of such measurements in clinical practice is limited. The introduction of the RARE pulse sequence was an important step towards the introduction of hybrid sequences which seem to be the key to rapid abdominal imaging. The introduction of functional magnetic resonance imaging ( $\mathrm{FMRI}$ ) and the use of MR to guide minimally invasive procedures may have an important impact, but at least fMRI so far has had much less of a clinical impact, than one might 
expect from the number of papers published in this field. The clinical impact leader of the last two years is clearly contrast media enhanced MR angiography.

Even in a complex technology like MR imaging, the relatively simple concepts have prevailed in clinical practice: these principles can be summarized easily:

'high contrast-high resolution-high speed'.

So, while MR imaging has not revolutionized crosssectional imaging, it has become very important in brain and musculoskeletal imaging, provides some good body imaging and, recently, good angiograms. On the other hand, there are important imaging needs not yet successfully covered by MR. Notable examples are: imaging of lung parenchyma, tumor staging, cardiac one-stop-shop imaging, metabolic imaging; and MR is still relatively costly. So, as in many fields of science, one notes a great discrepancy between the number of papers published in the field of MR and their clinical impact. Hence, when looking over the MR literature or reviewing a manuscript, in the mind of this author a statement keeps surfacing increasingly frequently, which may even become his reviewer signature:

\section{'So what!'}

The flexibility of MR imaging is in fact at the same time its virtue and its danger, much more so than with the other imaging modalities. So many things are possible by changing various parameters, that MR produces many great but also lots of irrelevant things. Care has to be taken that MR will not eventually find itself in a tower of Babylon situation (Fig. 2) where even people within the field do not understand each other any more. It is a firm belief of any scientifically trained person, that one should keep things as simple as possible, and MR can easily seduce us to get more and more complex. By looking back at the brief history of clinical MR imaging this appears to be true as well. An example for this contention is shown in Fig. 3. The MR researchers partly engaged in the basis of imaging have always been struck by the beauty of the physics MR angiography using time of flight and phase contrast methods, and look where these methods are now! Almost completely blown away by a very simple and eminently stable high speed-high resolution technique: contrast media enhanced magnetic resonance angiography (MRA).

\section{The six rules}

This leads me to formulate some rules where to look for clinically useful progress. Rule number 1 has precisely to do with keeping things simple. With four parameters you can fit an elephant and with six the fit is perfect! This is illustrated in Fig. 4, which shows the
'Fourier Elephant' [2], a free interpretation of Antoinede St. Exupery's famous drawings in the book 'The Little Prince' which is a literary must for every individual in this world [3]. Rarely we have the quality of data that multiparameter fitting with more than a few parameter yields reliable results.

Thus, if you engage in a project, where introduction into clinical practice will require multiparameter fitting and reliably detecting $2 \%$ changes, think twice before spending your energy. Things which are too complex to implement in a clinical environment often do not work well and are eventually replaced. As an example, Fig. 5 represents data of perfusion imaging of the heart using MR and PET, illustrating two basic principles of perfusion imaging: bolus tracking and wash-in experiments [2]. Finding an MR contrast agent which sticks to tissue in proportion to regional perfusion may be much more elegant than input deconvolution fitting, which currently has to be done to extract perfusion information from MR images. Nuclear medicine tracers used in imaging myocardial perfusion are wash-in agents, here ammonia rest and stress as well as glucose metabolism PET images. While MR perfusion imaging with input deconvolution may eventually consistently work, it would be a lot nicer to have a MR contrast medium which sticks for a long time in the heart like the nuclear medicine agents. Would such MR contrast agents be available, one could just use standard sequences for cardiac imaging to measure myocardial perfusion. Thus, rule number 1 is:

'Offer the clinician simple experiments, because with fo ur parameters you can fit an elephant.'

Rule number 2 is:

'Go for big effects of the order of $100-1000 \%$, they are always more reproducible and make the measurement stable, which is needed because the patient is usually unstable.' Contrast agent-enhanced MRA works so well because there is almost no signal other than that from the blood vessels, as illustrated in an example of a patient with aneurysmatic thigh disease (Fig. 6). Rule number 2 is at the same time a caveat for fMRI: it is a very interesting technique but signal changes are but a few percent. Hence, the method is technically demanding and 'the threshold of nonsense production is low'. This statement should not distract from the fact that fMRI has developed into a formidable research tool.

Rule number 3 concerns spatial and contrast resolution.

'A disease process is often $1 \mathrm{~cm}$ or less in size and should be readily detectable.'

Critical structures such as lymph nodes have this dimension, and this is indeed the technical limitation 


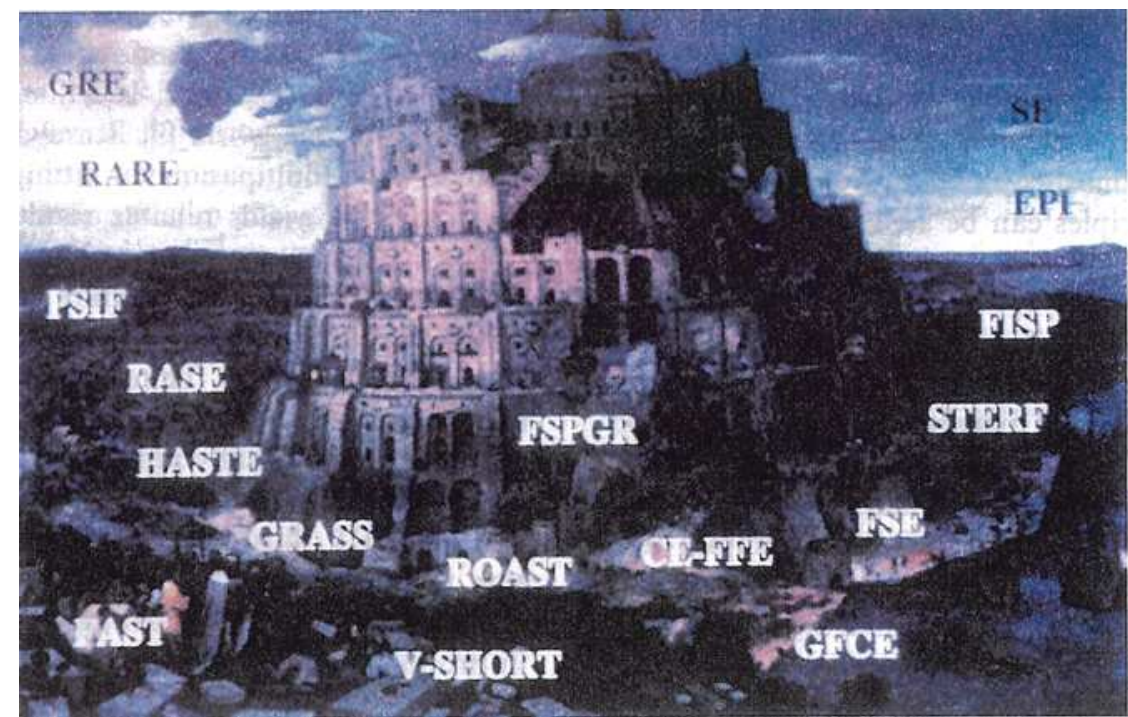

Fig. 2

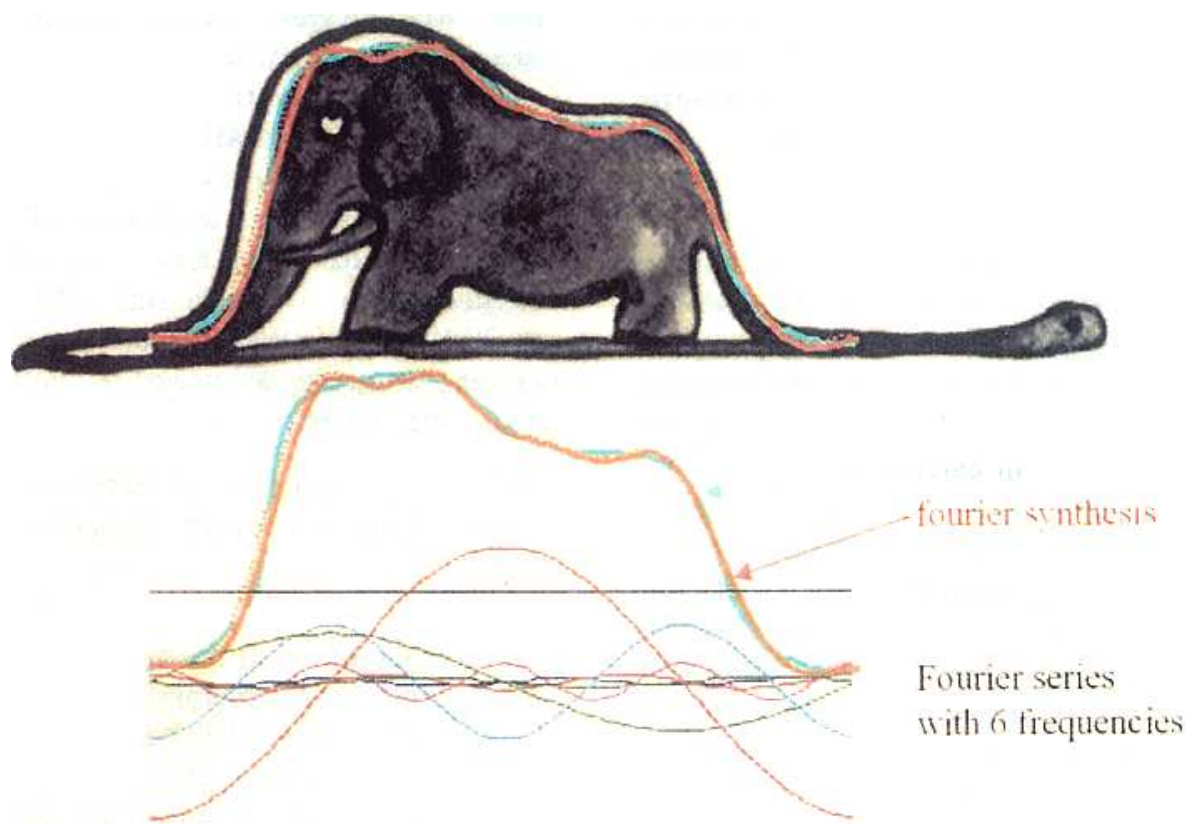

Fig. 4

Fig. 2. The Tower of Babylon; Pieter Breugel, the Elder, adorned with multiple abbreviations for pulse sequences. Allegory for potential communication problems within the MR community. The meaning of the abbreviations is defined for example in Ref. [5].

Fig. 4. The 'Fourier Elephant', redrawn after St. Exupery, showing that with four parameters one can fit an elephant and with six of them even well. This is to document that overanalysis of data will probably yield the desired results but they may be meaningless.

for clinical MR spectroscopy at this point. The small size of the pathology to be looked for and the inordinate amount of data in cross-sectional images makes morphological imaging clinically so challenging in this setting, particularly in tumor staging. This is illustrated by a page of a Richard Scarry children's book (Fig. 7) [4]. The task for a child is to find 'Lowly the Worm' in Fig. 7a in analogy to finding a lymph node or other small pathological structures on a large number of CT or MR sections (Fig. 7b). In this circumstance it is useful to have an imaging method which highlights the pathology with excellent contrast, and blurs irrelevant structures, so that the lesion lights up; spatial resolution is not everything, the contrast has to be appropriate.

This is illustrated in Fig. 8(a). In MR, classically this can be achieved to some degree by acquiring $\mathrm{T} 2$ weighted images, but there are many normal structures showing high signal intensity even on T2-weighted images. A method providing very high contrast at relatively good resolution is PET imaging, which in 
extended tumor staging seems to be the method of choice as illustrated on the right by a $3 \mathrm{~mm}$ melanoma metastasis.

Rule number 4 concerns good data acquisition:

'Where there is no data, there is no data.'

By milking poor data, little additional substance can be produced. Postprocessing is only useful when the data set on which you run the postprocessing algorithms are sound. There are some very elegant filtering techniques able to cull information from temporal data series such as auto- and cross correlation analysis used in the postprocessing of fMRI images [5], but there the data is hidden in a series of images not obvious to the observer. However, stacks of images in a $3 \mathrm{D}$ data set with poor signal-to-noise ratio will not get better by filtering, just more pleasing to look at. The very high signal-to-noise ratio is the reason why contrast media enhanced MRA is so good; there is lots of good data in an MR angiographic image.

Rule number 5 relates to contrast media enhancement in MR:

'MR contrast agents act at concentrations of $10^{-4}$ $10^{-5} \mathrm{~mol}$, receptor interactions occur typically at $10^{-9}-10^{-12}$ mol.'

The consequence of this observation is, that MR contrast agents cannot be expected to produce enhance-

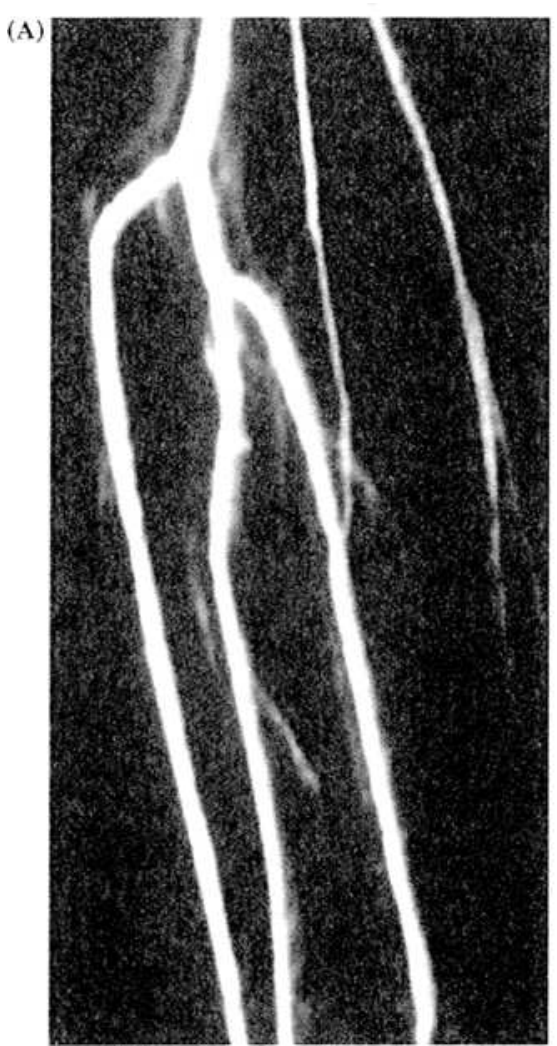

ment in all desirable settings. Specifically, processes which occur in the nano- to picomolar range, for example dopamine receptor interactions as shown in a PET scan (Fig. 9) cannot be visualized by MR imaging. MR signal changes occur only when contrast-active substances are present in the micromolar range, such as is the case with blood pool markers.

Rule number 6 is simple and an old wisdom with all imaging specialists who have been around for a while:

'Faster is better.'

Whenever high resolution images can be acquired faster than slower, the methodology is bound to prevail because motion artifacts disappear. This is again well exemplified with contrast media enhanced MRA, where next to the good contrast the fact that data can be acquired in a breath hold, has made this technique to become so successful.

\section{Predictions}

Using these rules we can now start predicting where MR may be in the year 2010 . The six rules provide a base. Before trying to make some predictions, it has to be reiterated that we cannot predict 'revolutionary' changes in imaging because some new technological

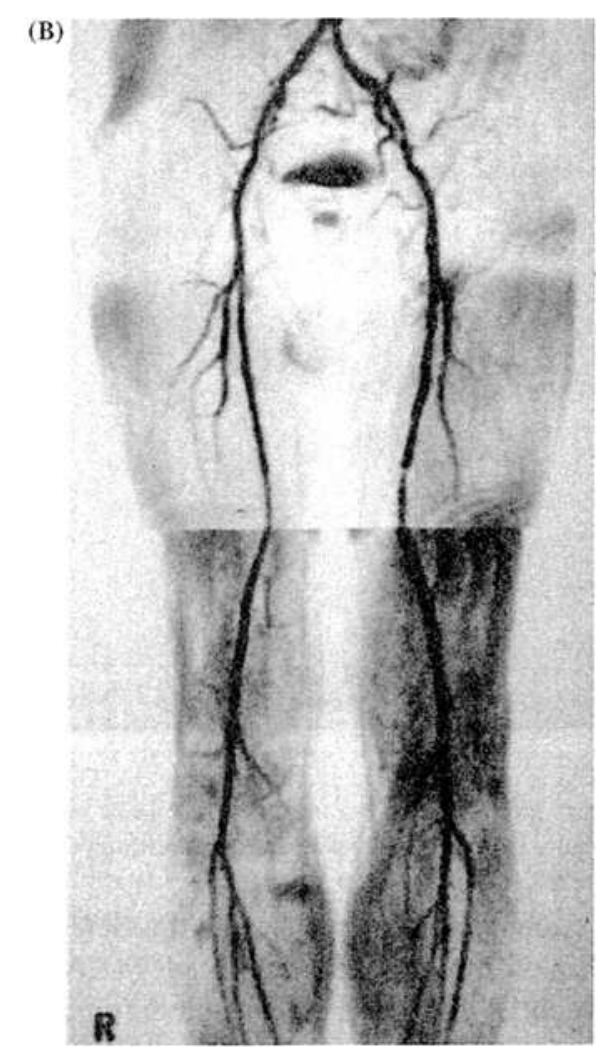

Fig. 3. Time-of-flight MRA (A) and contrast agent-enhanced MRA (B) demonstrating the superior quality of modern contrast agent-enhanced MRA. 
be used only for regional tumor staging and have little impact on imaging of metabolism and for tissue specification. Quantitative MR imaging beyond flow will be difficult mainly because of the difficulty to quantify MR signals in general.
Finally, what was stated emphatically at the beginning, will have to be reiterated at the end of this treatise. All the conjectures made here may be wrong because any number of unpredictable 'revolutionary' inventions of any of the readers of this text, or possibly

(a)
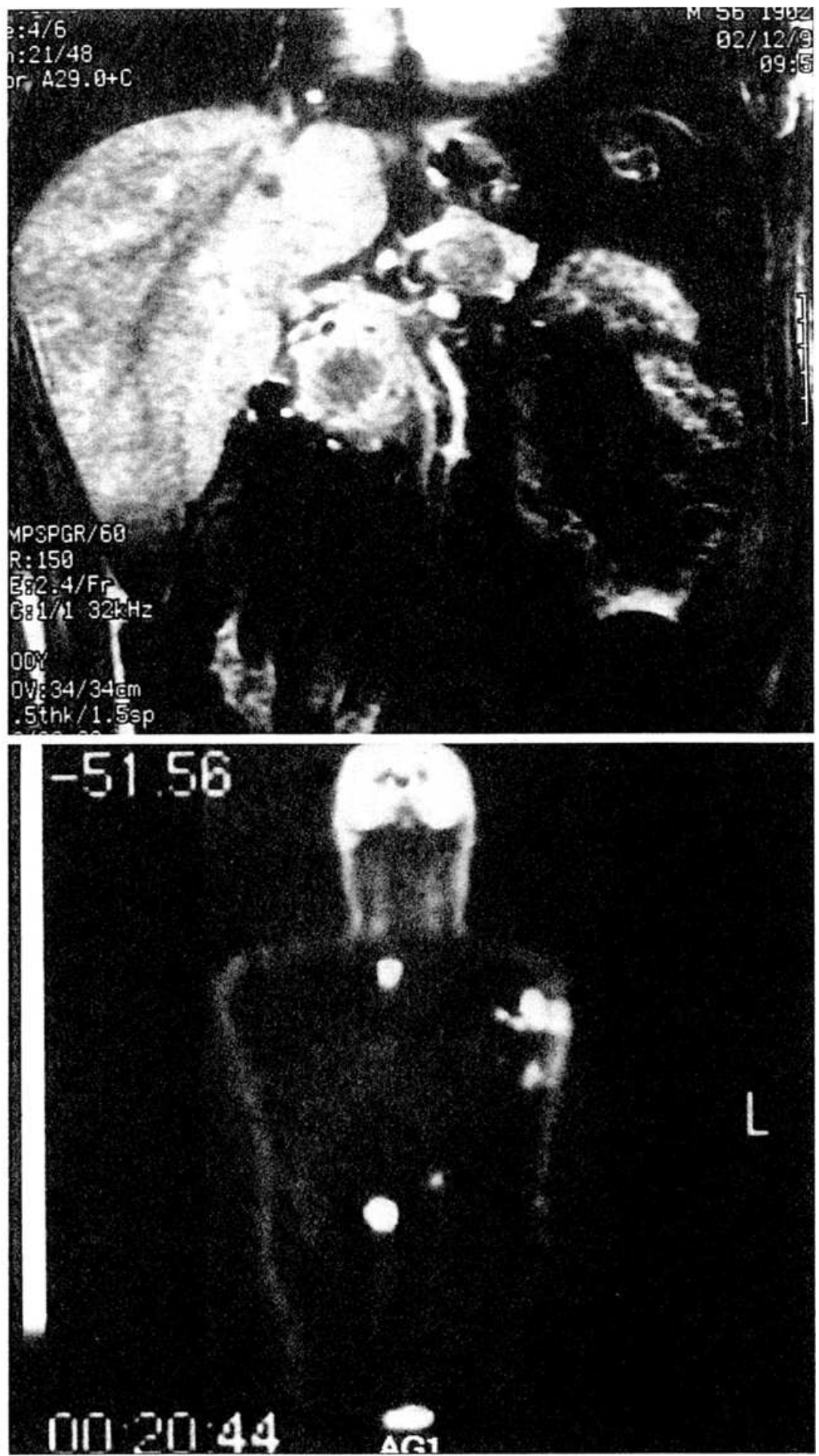

Fig. 13. Patient with a pancreatic carcinoma showing (a) local disease on MR imaging and (b) extended disease on a corresponding coronal whole body PET scan section. 


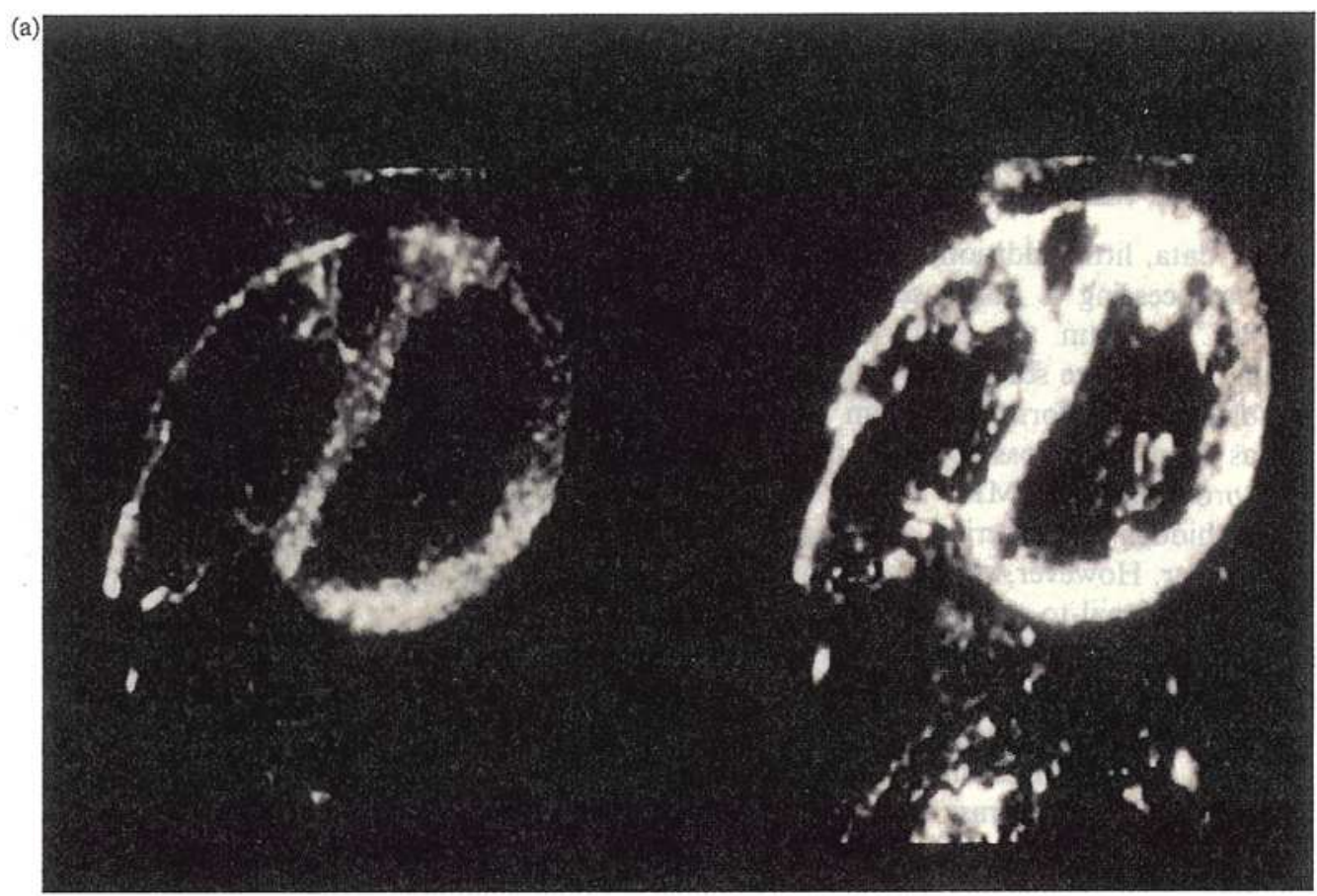

(b)
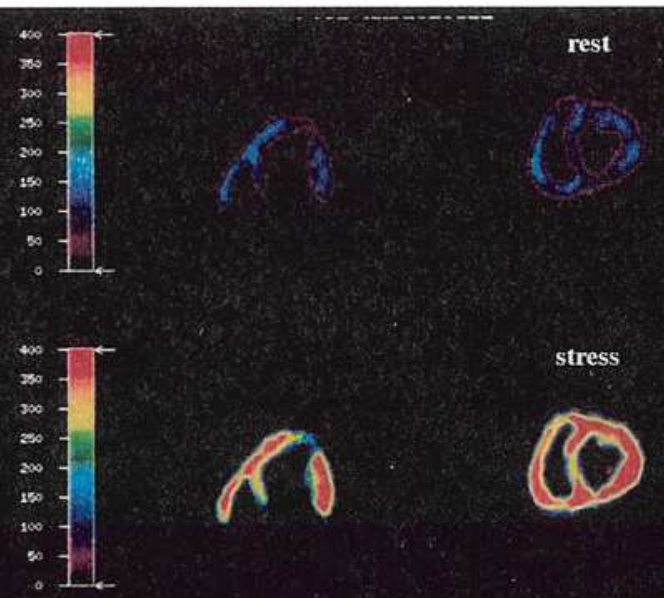

stress
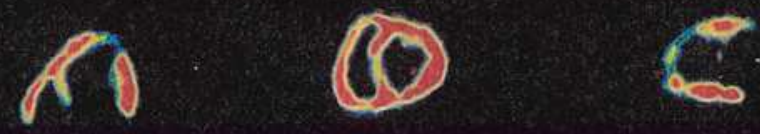

coronary reserve
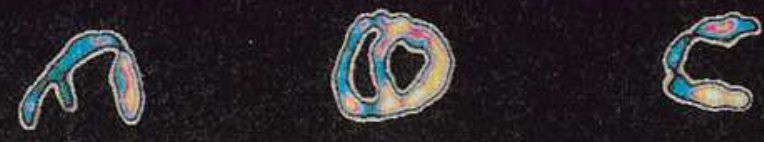

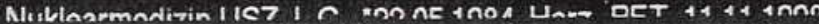

Fig. 5. MR perfusion imaging (a) and PET perfusion imaging (b). The former shows relatively good spatial resolution on this two-shot echo planar image pre- (left) and approximately $30 \mathrm{~s}$ after injection of contrast media (right), while the latter shows rest and stress ammonia PET images with good contrast and a potential for relatively simple quantification. The bottom row depicts pixelwise perfusion reserve.

development may suddenly make much work obsolete, as has occurred with contrast-agent enhanced MRA doing away with much of the other MR angiographic techniques.

Brain imaging in the year 2010 will no longer make use of CT in most cases. Morphology, early stroke detection and even emergency trauma cases will be done in MR. fMRI will be a useful adjunct mostly during brain surgery, but will have no substantial impact on understanding affective disorders such as de- 
pression. This is because affective disorders and schizophrenia are more likely related to neuroreceptor derangements. fMRI will thus remain an interesting research tool, but plateau earlier in its clinical applications than many of us expect because of the limitations of the method and the complexity of the brain.

Morphological and functional brain data will be fused on a regular basis and integrated systems combining modalities like $\mathrm{CT}$ and PET may emerge, and real-time fusion of interventional $\mathrm{MR}$ and $\mathrm{CT}$ with PET data during brain surgery may become standard. Complex tumor surgery will occur to a large extent in open MR units, as identification of tumor margins is considered to be of major importance for successful disease control. Smaller dedicated head units will make brain MR imaging more cost effective.

In cardiovascular imaging, contrast enhanced MRA will have replaced much of vascular angiography by 2010, a process which has started already now in all institutions with high end MR scanners.

With the advent of blood pool contrast agents, optimization of data acquisition will be accomplished so that MRA can consistently visualize the coronaries and diagnose stenoses in such a large fraction of patients, that MRCA will show clinical utility. Since visualizing the coronaries is the major driving force to make MR imaging of the heart relevant and cost effective, the other cardiac MR techniques, i.e. cardiac anatomic imaging, wall motion imaging, MR imaging of cardiac volumetry and even perfusion imaging which may remain complex, will be used together with MRCA to make cardiac MR the long asked for 'one-stop-shop' heart examination, which cardiac MR specialists have been talking about for the last decade.

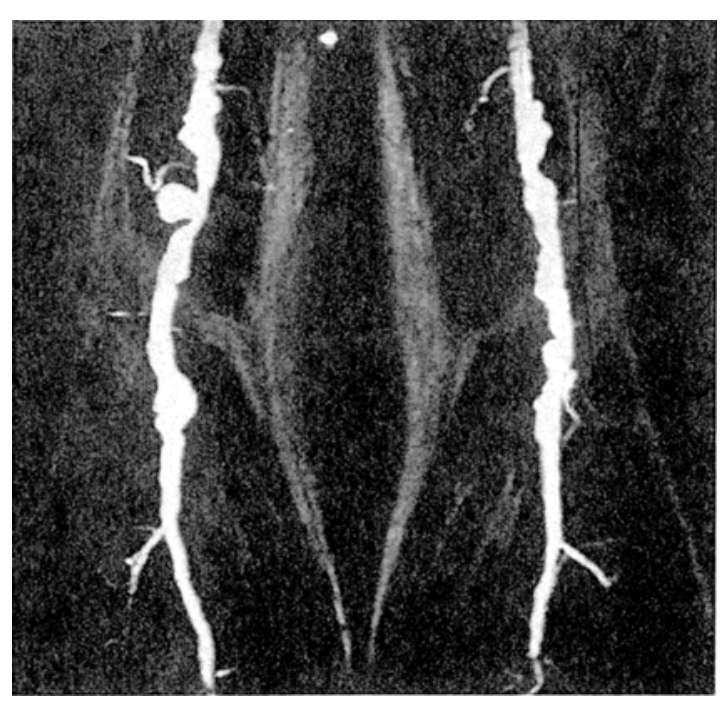

Fig. 6. Contrast enhanced MRA of a patient with aneurysmatic disease of the arteries of the lower extremities.
It is doubtful for toxicity reasons but not impossible, that a wash-in agent, i. e. an agent sticking to the myocardium in proportion to regional perfusion, will become available as MR perfusion agent. If such an agent would indeed be available, perfusion imaging as currently used in nuclear medicine can be done with MRI which would make MR perfusion imaging in itself a method very competitive with thallium and Tc-MIBI/ tetrofosmin imaging due to the much better spatial resolution obtained with $\mathrm{MR}$ imaging.

More complicated methods like myocardial tagging will unlikely enter clinical routine as no impact on management of disease has been derived from this methodology at this point, although tagging may remain an important research tool. Metabolic imaging of the heart with MR spectroscopy will not enter clinical routine because of the lack of spatial-temporal resolution. On the other hand, it is reasonable to assume, that many cardiovascular interventions will be done under MR guidance in the year 2010 , not only because of the lack of ionizing radiation to the physician, but because of the added value of 3D imaging, plaque characterization and flow measurements to control results. Fig. 10 depicts key frames in a percutaneous transluminal angioplasty (PTA) performed under MR guidance. Fig. 11 depicts a MR 'road map' with two instrument tips superimposed in real time over it. Fig. 12 is an example of intravascular imaging with anatomic correlation using a balloon catheter fitted with a local coil. As understanding plaque composition is important, intravascular MR imaging during an intervention will become routine and the tools to support intravascular imaging and intravascular interventions are being developed. It has to be noted that endovascular MR imaging is highly competitive to endovascular ultrasound because the cost of the probes is going to be much lower.

In the gastrointestinal and genitourinary systems MR will take over most CT indications by 2010 and become the major morphological staging method in the abdomen and pelvis. This is a result of the better inherent contrast, the contrast material, and the hybrid pulse sequences now affording breath-hold high-quality images of the abdomen.

In the musculoskeletal system $\mathrm{MR}$ will cement its role as prime morphological imaging modality in the next decade. In the extremities, cheap units will become widespread and MR will be used as screening modality for back pain. Functional examinations will become relevant as more open $\mathrm{MR}$ units become available. Furthermore, MR guided surgery will become commonplace in musculoskeletal imaging. Thermoablation of tumors such as lumbar disk disease, soft tissue tumors, etc. will be done under MR control.

General predictions are the following: short-bore high-field MR scanners will become available not only 


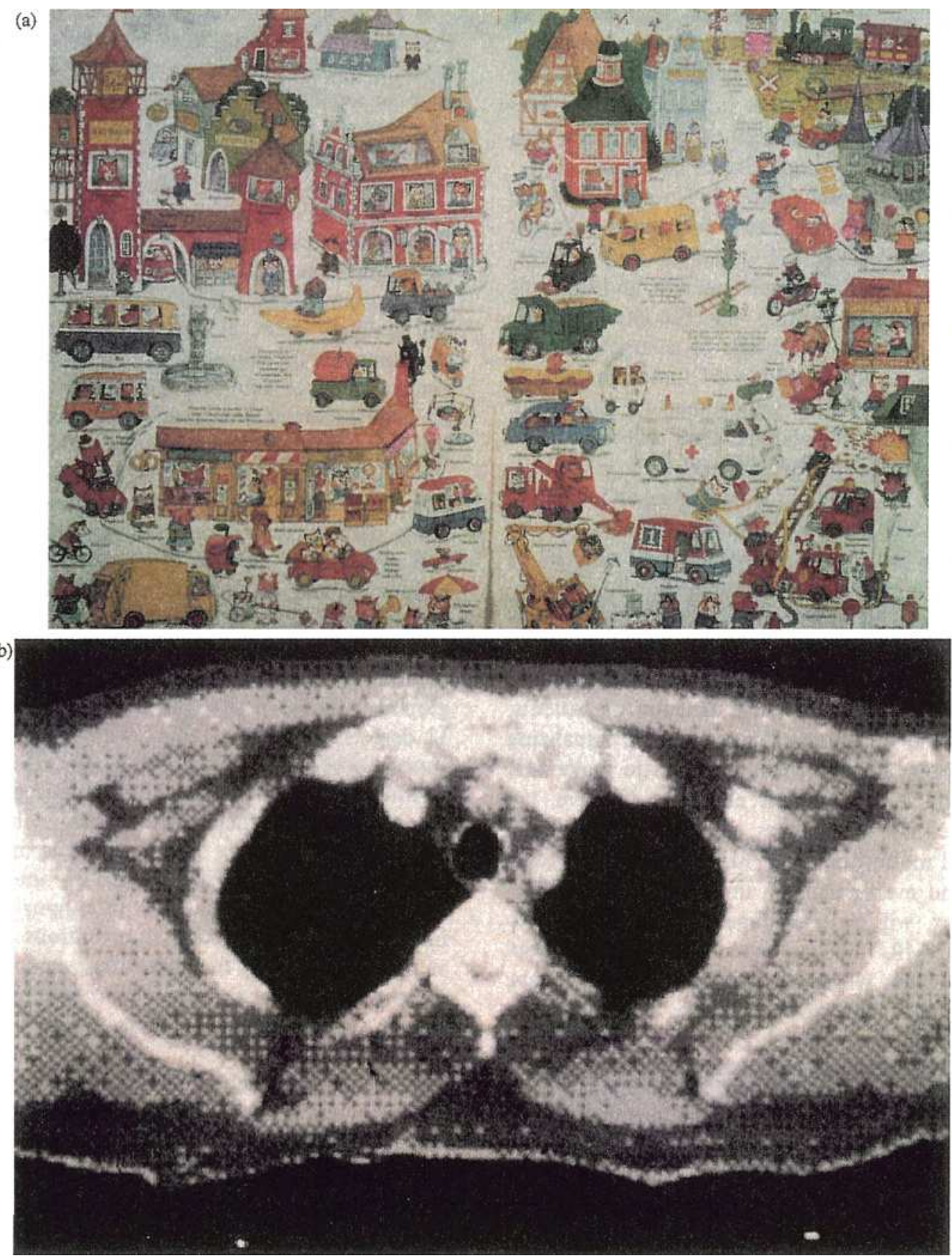

Fig. 7. (a) A page from a Richard Scarry children's book depicting 'Lowly the Worm' sitting in an apple mobile (arrow); (b) CT scan showing a small possibly pathological structure in the subcutaneous tissue of the right posterior thoracic region, which was prospectively overlooked in a stack of 40 sections (arrow).

for imaging but for interventions, mainly vascular interventions such as stenting, which will be routinely done under MR guidance in view of excellent vascular contrast, but also contrast against the surrounding soft tissues in all vessels except the coronaries where spatial resolution may still be a limiting factor for guiding interventions, but not for depiction of anatomy. There will be widespread use of open MR systems for mini- mally invasive surgery, and miniaturization technologies will bring forth MR compatible mini-devices useful in measuring MR signals anywhere from within the body which can even be left in place for future MR measurements.

The author does not foresee a substantial increase in the role of metabolic MR imaging, and the pharmacology and physiology of producing MR contrast media 
which can label physiological processes beyond vascular and RES enhancement may be so prohibitively difficult, that not too many agents will be available with such properties by 2010 . While MR will be the primary morphological tumor staging modality to evaluate local disease spread, PET will be the method of choice for whole body staging to screen whether the tumor has spread to distant sites (Fig. 13).
Investment strategies concerning high technology medical imaging equipment therefore should be as follows. University hospitals and large clinical centers will have to further invest heavily into MR and the number of MR units will have surpassed that of CT scanners and outnumber them by at least 2:1. While standard magnets will still be in use, more dedicated units will have to be purchased: head units, extremity units, open

(a)
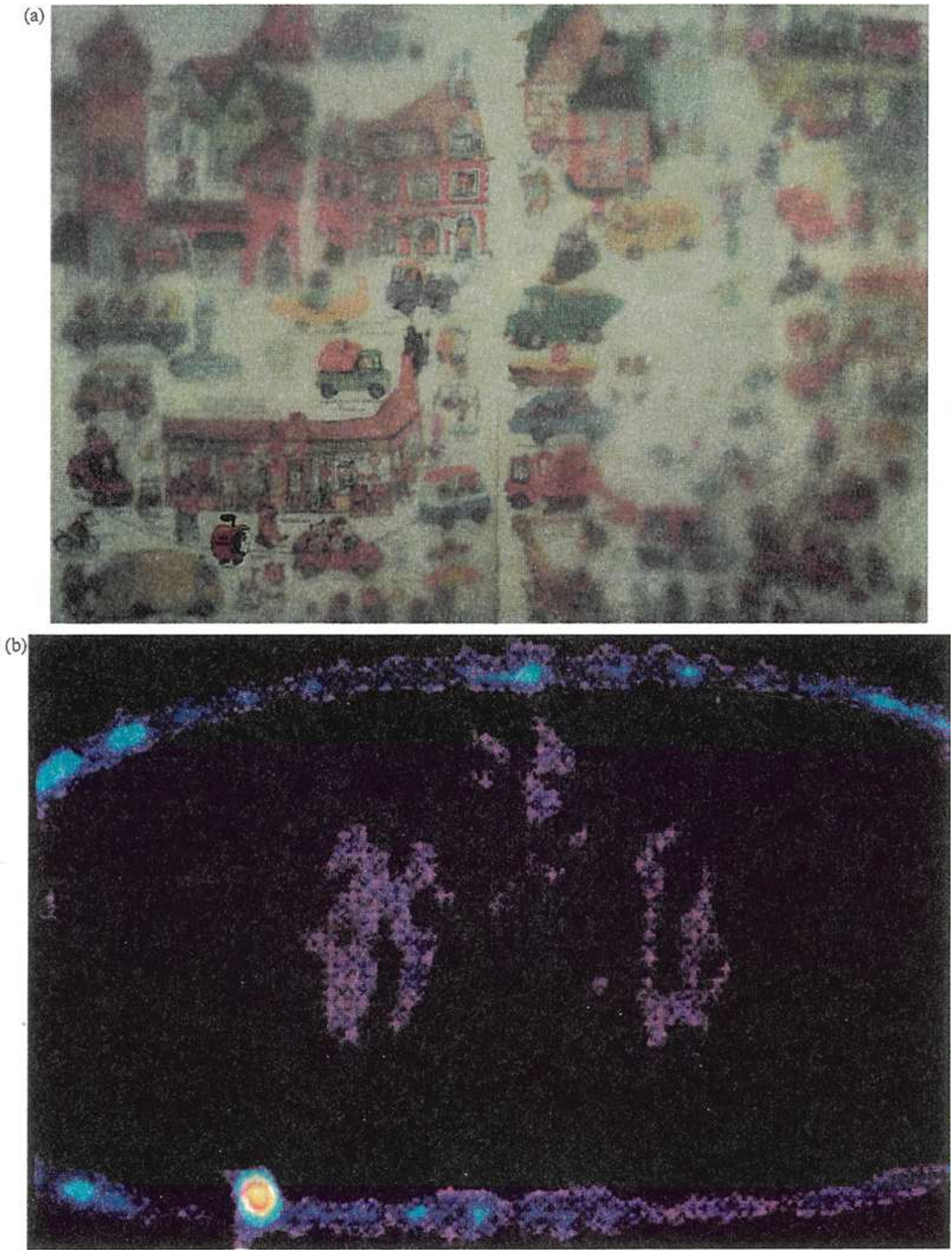

Fig. 8. (a) Same page from the children's book shown in Fig. 7(a), but now all structures except that containing 'Lowly the Worm' are blurred; thus, much more contrast is available. In tumor imaging, this is exemplified by a PET scan demonstrating a 3 mm melanoma metastasis. 


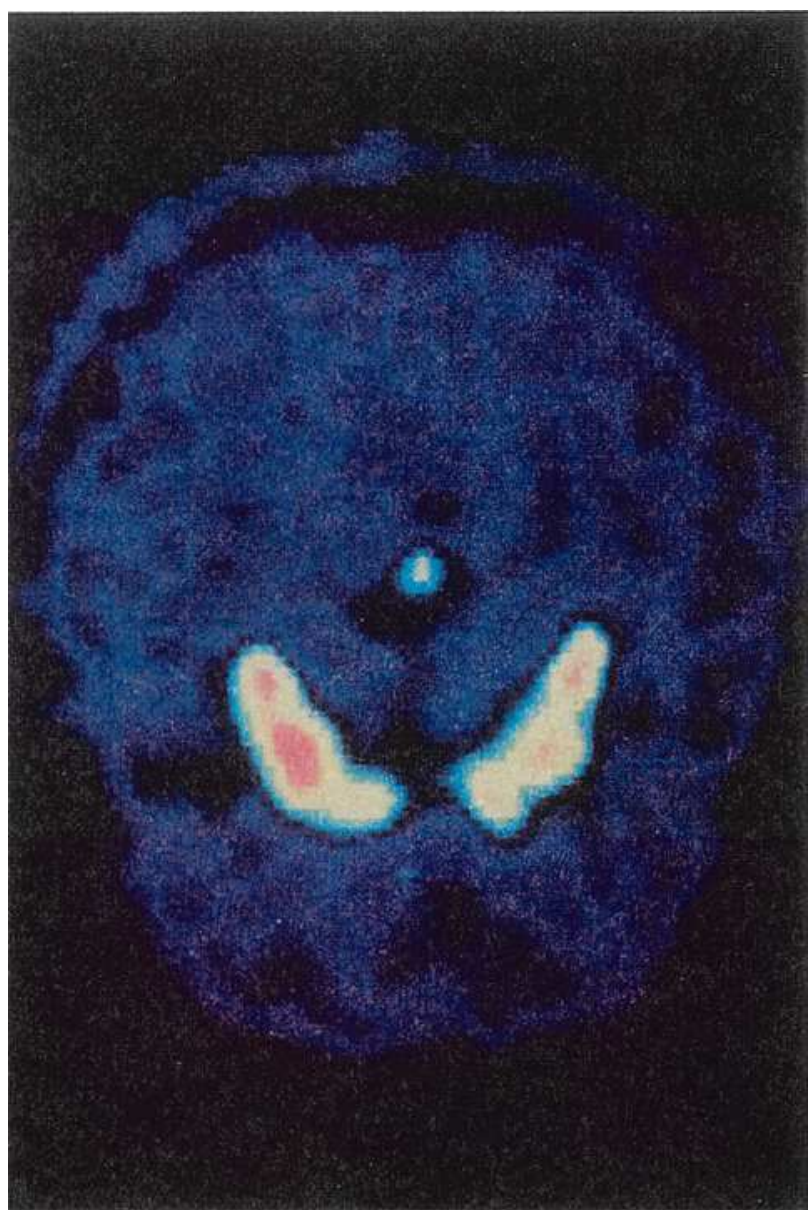

Fig. 9

\section{MR guided PTA}

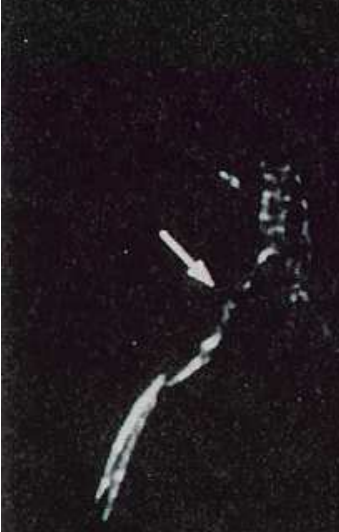

pre PIA

\section{Gdilled Balloon}

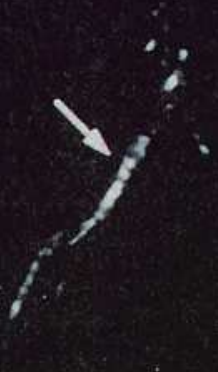

Last BIA

Fig. 10

Fig. 9. PET scan using a dopamin receptor marker (F-18-DOPA) showing the caudate and striatum only.

Fig. 10. PTA (percutaneous transluminal angioplasty) of an iliac stenosis pre, during and after balloon angioplasty, monitored with a $0.5 \mathrm{~T}$ interventional open magnet (note that this MR imager configuration and field strength are not optimal for this purpose and short bore, high field magnets will be used. 


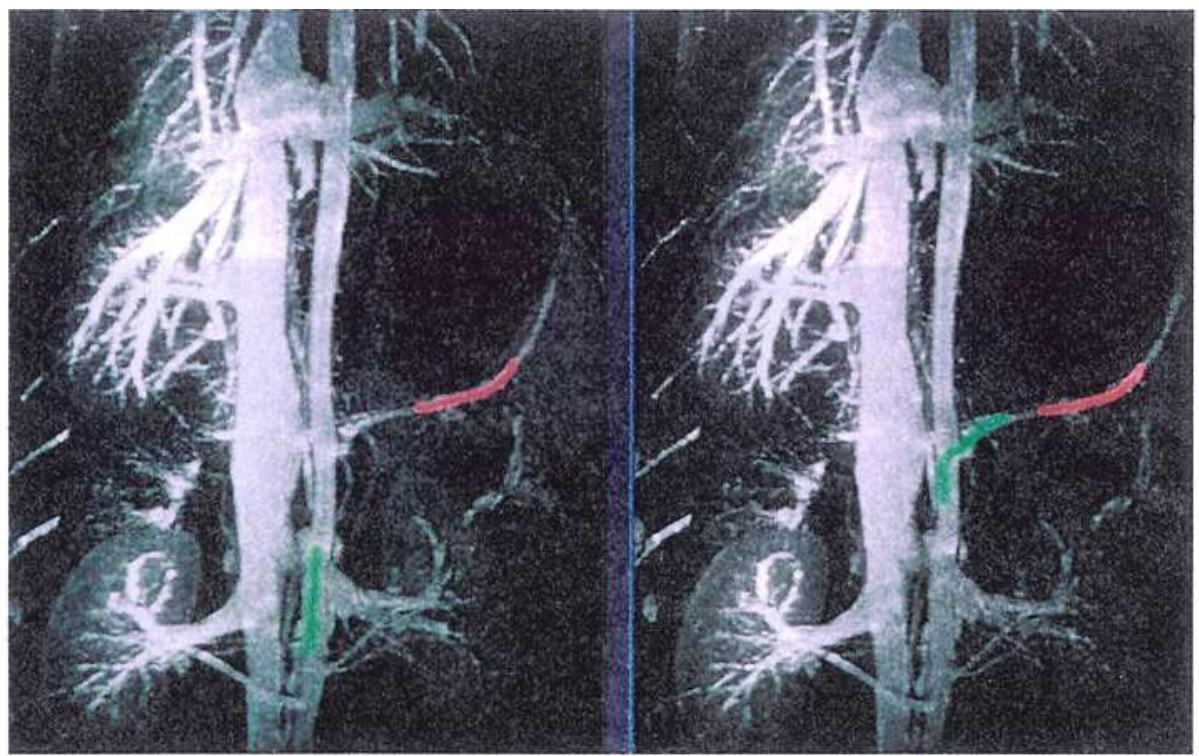

Fig. 11

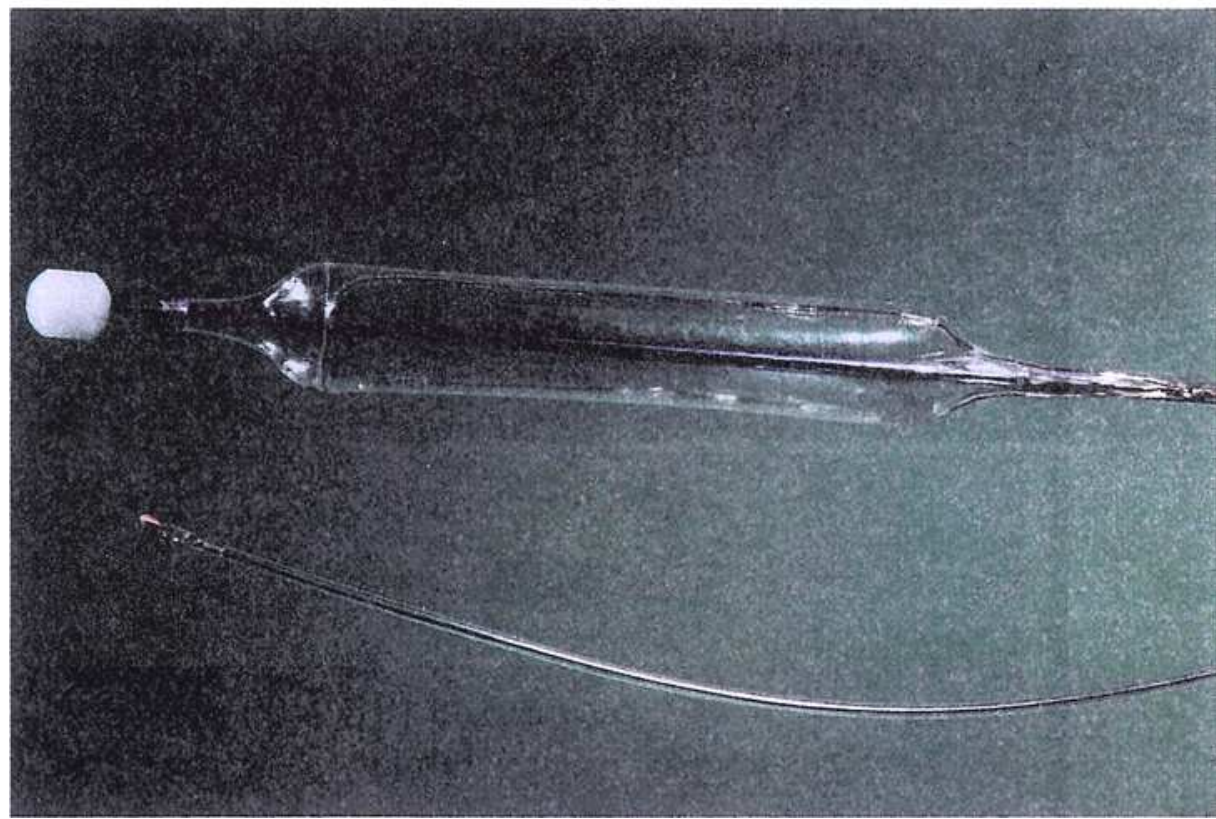

Fig. 12

Fig. 11. Road map produced with contrast media enhanced MRA with the superposition of two instrument positions (Courtesy of Dr M. Ladd). Fig. 12. Intravascular imaging with a local coil fitted onto a balloon catheter which is inflated during imaging.

systems for performing surgery and short bore systems as general units or units providing also for interventional procedures. Investment into $4 \mathrm{~T}$ and beyond will still be mainly for research rather than clinical purposes.

University centers will also have to install PET centers as PET will become the major tumor staging modality for extended disease. For smaller centers, specialized MR units will still be necessary because they are cost effective when the volume is large enough. Expensive open units for surgery and high field units will probably not spread to such sites.

In conclusion, I predict that MR imaging will be the most important diagnostic imaging modality in 2010. Many interventions will be guided by MR using new virtual reality possibilities and microelectronic concepts. Furthermore, MRI will be substantially cheaper than today due to the large scale introduction of dedicated smaller units. Even MRI has technical limitations and may stay inferior in some imaging areas. MR will remain inferior in imaging the lung parenchyma and compact bony structures, where CT will prevail. It will 
of somebody not working in the field of MR, may change the outlook in the clinical environment of $M R$ to such an extent, that all the premises on which the predictions made here are based, have become obsolete. Thus, it is important that we remain open to ideas of others and to ideas surfacing in other neighboring fields of knowledge, even looking occasionally beyond the imaging horizon, as for example at the economic environment which will probably very heavily affect our clinical work in the next 10 years.

\section{Acknowledgements}

The author wishes to acknowledge his collaborators who have substantially contributed to this overview.
They are, in alphabetical order: Jörg F. Debatin, MD, Benjamin Dubno, MD, Paul Hilfiker, MD, Mark Ladd, $\mathrm{PhD}$, Graeme McKinnon, $\mathrm{PhD}$, Thomas Pfammatter, MD, Gesine Paul-Zimmermann, MD, Paul Steiner, Hans Steinert, MD and Simon Wildermuth, MD.

\section{References}

[1] Kuhn T. The Structure of Scientific Revolutions. Chicago, IL: University of Chicago Press, 1994.

[2] von Schulthess GK. Hennig J, editors. Functional Imaging. Philadelphia, New York: Lippincott, Raven, 1998.

[3] St. Exupery A. The Little Prince. London: Heinemann.

[4] Scarry L. The Adventures of Lowly the Worm. New York: Random House, 1995.

[5] Petterson H, Allison D. In: Smith HJ, von Schulthess GK, editors. The Encyclopedia of Medical Imaging, vol. I. Oslo/London: The NICER Institute/ISIS, 1998. 